\title{
STUDY OF PASSIVE VIBRATION ASSISTED ROTARY DRILLING TOOL WITH THE HELP OF BOLD GRAPHS
}

\author{
Dipesh Maharjan ${ }^{1}$, Jeronimo De Moura Junior ${ }^{2}$, Geoff Rideout $^{3} \&$ Stephen Butt $^{4}$ \\ 1, 2, 3, ${ }^{4}$ Faculty of Engineering and Applied Science \\ Memorial University of Newfoundland, St. John's, NL, CA \\ dmaharjan@mun.ca ${ }^{1}$,jdemourajuni@mun.ca ${ }^{2}$, g.rideout@mun.ca ${ }^{3}$, sdbutt@mun.ca ${ }^{4}$
}

\begin{abstract}
The use of passive-Vibration Assisted Rotary Drilling (p-VARD) tool to improve drilling performance has been experimented and well documented in many occasions. In addition to increasing the Dynamic Downhole Weight on Bit (DDWOB), the use of this tool has also reported in improvement in the drilling rate of penetration. The works until now are limited to experimental studies and lack the explanation as to how it actually works. Theoretically, adding a p-VARD tool increases the compliance of the drill string in an axial direction allowing the drill bit to oscillate axially. In this paper, a mass-spring-damper bond graph model of p-VARD tool is built in 20-Sim. The spring is modelled with the help of AlmenLaszlo's load-deflection equation for Belleville springs and the damper is modelled as neoprene rubbers with moderate damping ratio. The bit-rock interaction is represented in the form of a source of flow providing sinusoidal displacement to the drill bit and the oscillating portion of the p-VARD tool. A parametric analysis of the drilling system consisting of a $\mathrm{p}$ VARD tool shows that the resonating frequency of the system can be lowered to a workable range with the help of this tool thus allowing it to resonate and amplify the impact generated. Similarly, this modeling tool also enables us to study the parameters which may flatten the Belleville springs. A stack of flat springs is as stiff as the rigid drill string and this terminates the impact generated by the resonance. Results show that spring and damper inside the p-VARD tool can be tuned to be operated at different Weight on Bit (WOB) and excitation parameters.
\end{abstract}

Keywords-drilling optimization, bond-graph, p-VARD, DDWOB

\section{INTRODUCTION}

Prediction and optimization of drilling rate of penetration has been the center of numerous research works for a long time in the drilling industry. The perfect hole cleaning model by Maurer in 1962 [1] is one of the oldest and the most widely used models to predict the Rate of Penetration (ROP) as a function of Weight On Bit (WOB), rotary speed, formation strength and bit diameter. Apart from this, other researches have also produced models to predict ROP [2] and Mechanical Specific Energy (MSE) [3] or Hydromechanical Specific Energy (HMSE) [4]. The goal of all these research works is to enable the driller to predict effects of various drilling parameters on the ROP and optimize them for increasing the drilling efficiency.

With the goal of optimizing the drilling performance, a stream of research is focused in harnessing the vibration to improve the ROP. As a part of ongoing research, the study of vibration assisted rotary drilling has been one of the major focuses at the Drilling Technology Laboratory (DTL) of Memorial University of Newfoundland (MUN). In an experimental study by $\mathrm{Li}$ et al. [5] variation in vibration amplitude was found to be a significant factor in increasing the ROP using a coring bit. Similarly, Babatunde et al. 2011 [6] carried the work forward by investigating the effect of changing frequency and amplitude of vibration. Both of these experiments used an electromechanical axial shaker mounted at the bottom of the drill stand as the source of active vibration and demonstrated impact of vibration in enhancing the drilling performance.

In another set of experiments performed by Babatunde et al. 2011 [6], the effect of change of vibration parameters was studied on a diamond drag bit which showed significant increase in ROP at controlled frequencies. Experiments with Polycrystalline Diamond Compact (PDC) bits show that with the compliance tool low WOB cannot produce a drastic improvement in the ROP as the main bit cutter face cannot partake in penetration process [7]. One of the hypotheses that has been proposed in this research is that axial compliance in the drill string creates the bit rock displacement which reduces the cuttings accumulation in the zone of penetration thus optimizing bottom hole cleaning [7].

Numerous lab and field scale experimental works with the pVARD tool have demonstrated improvements in the drilling performance. In a recent experimental work involving the p-VARD tool, Molgaard et al. [8] reported a significant increase in DDWOB with the use of p-VARD tool. The experiments involved drilling holes parallel to shale bedding on specimens of equal height using a dual cutter PDC and diamond impregnated coring bit. The DDWOB is the WOB measured by the load cell placed below the drilling specimen. Similarly,

This work focuses on development and analysis of bond graph model of p-VARD tool. The numerical model developed with the help of the bond graph enables us to see the 
relationships between different parameters and guide us towards how to best optimize them for enhancing the drilling performance. A comparative study has been performed to illustrate how the use of $\mathrm{p}$-VARD tool dampens the vibration at the drill rig while optimizing the DDWOB.

\section{MODELING OF DRILLING SYSTEM}

The drilling system analyzed in this paper is a Small Drilling Simulator (SDS) at DTL, MUN, the details on which are given by Khorshidian et al. 2014 [9] and Shah et al. 2019 [10]. For simplicity, the sub-components of SDS that are included in the modeling are WOB, drill string/bottom hole assembly, formation stiffness and bit-rock interaction along with the $\mathrm{p}$ VARD tool. These sub-components make up the compliant and the stiff drilling system when operated with and without the pVARD tool. Figure 1 represents a schematic of the stiff drilling system, whereas Figure 2, with an addition of p-VARD tool, represents a compliant drilling system.

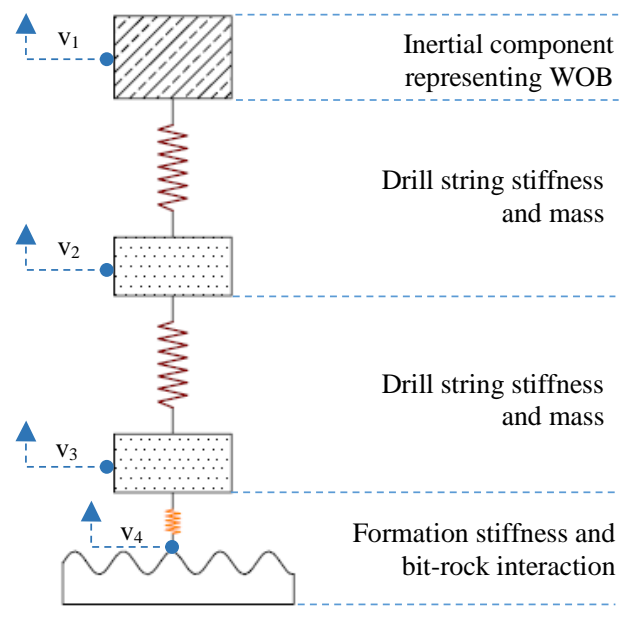

Figure 1: Schematic of stiff drilling system

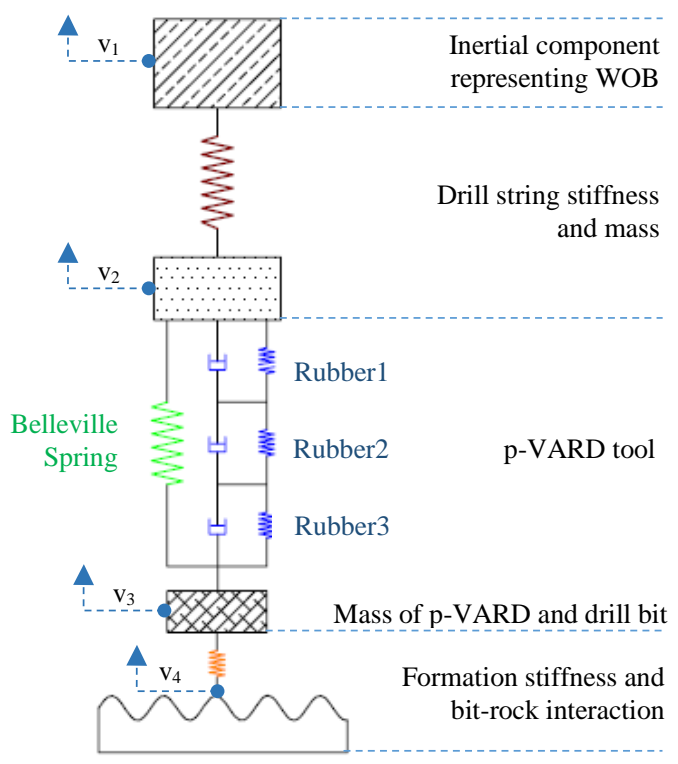

Figure 2: Schematic of drilling system with p-VARD

\section{A. Weight on Bit}

SDS transfers WOB from the suspended plates to the formation with the help of a rack and pinion mechanism. Additionally, WOB is generated by the weights of the mechanism, drill motor and swivel, which sits on the top of the drill string. For this reason, the WOB considered for the simulation is the weight of all these components combined, which is represented by a single inertial component in the schematic diagram. A general representation of the weight on bit that is applied to the systems is given by below equation.

$$
\begin{gathered}
\text { WOB }(k N)=a+b \times \text { number of plates }(n) \\
\text { where } a=0.8313 \\
b=0.1487
\end{gathered}
$$

\section{B. Drill String}

Drill string (DS) connects the SDS swivel to the drill bit and transfers torque, WOB and drilling fluid. A lumped parameter model of DS for axial motion is used to define the governing equations and it comprises of following parameters:

$$
\begin{aligned}
& \text { Stiffness }(k)=\frac{A E}{l}, \quad \text { Mass }(m)=\frac{\text { mass of } D S}{3} \\
& \text { where } A=D S \text { cross sectional area } \\
& E=\text { Young's Modulus } \\
& \text { and } l=\text { length of the } D S
\end{aligned}
$$

In addition to the stiffness, the DS is modelled with a damping ratio of $\zeta=0.01$, which is the standard value for small diameter steel pipes [11].

For this study, a 1 inch outer diameter (OD), 0.75 inch inner diameter (ID), 12 inch long DS is used to connect the inertial WOB component to the rest of the p-VARD drill string system.

\section{C. $p$-VARD tool}

This tool provides axial compliance and dampening to the vibration generated by the top drive system and the bit-rock interaction, while functioning as a part of the drill string. In Figure 2, the p-VARD tool is represented as a parallel combination of Belleville springs and rubbers, whose characteristic properties are presented in following sections.

\section{1) Belleville springs}

The p-VARD tool uses Belleville springs as an energy storage device. High strength and flexible compliance make these springs suitable for the p-VARD tool. Although many researches have been done to plot the load-deflection characteristics of these springs, the first equation proposed by Almen-Laszlo [12] is used for the purpose of this research because of its simplicity. Relations proposed by Curti and Montanini [13] include the effect of edge friction and that by Ozaki et al. [14] include the effect of edge and surface friction. Similarly, in one of the works done by Shah et al. 2020 [15], different stacks of Belleville springs used in the p-VARD tool have been experimentally tested and the load deflection curve, which is generated is tested against the numerical models.

The unique nature of the Belleville spring stack make it necessary to model this component as a conditional compliant 
element. When the spring is fully compressed, it becomes as stiff as the DS and when it is relaxed beyond the uncompressed free height, it doesn't exert any pulling force.

Below equations, as proposed by Almen-Laszlo [12] are used to govern the load-deflection relation of the Belleville spring stack:

$$
\begin{aligned}
& P=J P_{m} \\
& \text { where } P_{m}=\frac{E \bar{\delta}}{\left(1-\vartheta^{2}\right) r_{2}^{2}}\left[(h-\bar{\delta})\left(h-\frac{\bar{\delta}}{2}\right) \frac{t}{M}+\frac{t^{3}}{N}\right] \\
& \frac{1}{M}=\left[\frac{\gamma+1}{\gamma-1}-\frac{2}{\ln \gamma}\right] \pi\left(\frac{\gamma}{\gamma-1}\right)^{2} \\
& \frac{1}{N}=\frac{\pi}{6} \ln \gamma\left(\frac{\gamma}{\gamma-1}\right)^{2} \\
& \gamma=\frac{r_{2}}{r_{1}}, \quad \bar{\delta}=\frac{\delta}{I}
\end{aligned}
$$

For the purpose of this study, a spring with 0.755 inch ID, 1.5 inch OD, 0.045 inch thickness $(\mathrm{t})$ and 0.093 inch height $(\mathrm{H})$ is chosen. Detail specification of this spring can be found under McMASTER reference number 9712K86.

\section{2) Rubber elements}

The rubbers used in the p-VARD tool serve the purpose of dampening the vibrations. Schematically, they are connected in parallel to the Belleville springs, but intrinsically, they are each composed of ideal spring and damper connected in parallel (see Figure 2).

An empirical relation can be established through a series of experiments to relate the stiffness and dampening coefficients of the rubbers to a range of excitation frequency and amplitudes. One such work performed by Zhong [16] produces equations for the stiffness and damping coefficients of field scale p-VARD rubbers. The field scale p-VARD tool uses 2.1 inch ID, 4.75 inch OD \& 0.5 inch thick Buna-N, also known as acrylonitrile butadiene rubber. These material properties are responsible for inherent stiffness of the rubber rings in addition to its damping, which is very challenging to be numerically modelled.

The lab scale p-VARD tool uses 1.5 inch ID, 2.3 inch OD \& 0.25 inch thick neoprene rubbers with moderate damping ratio

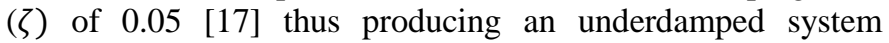
response. The closed cell characteristics of this material makes it suitable for using in lubricated environment. For the purpose of this study, the p-VARD rubbers are assumed to be ideal dampers with negligible stiffness. The following set of equations is used to determine the damping coefficient $(b)$ of the rubber stack.

$$
\zeta=\frac{\text { damping coefficient }}{\text { critical damping coefficient }}=\frac{b}{2 \sqrt{k m}}
$$

where $k$ and $m$ are equivalent stiffness and mass of the drill string model

A series connection of $n$ number of these rubbers would result in equivalent damping coefficient of $b / n$, which is input into the bond graph model to represent the rubber damping.

\section{Bit-rock interaction}

Bit rock interaction is reviewed in the literature as being a critical input for drill string vibration analysis. In the modeling field, bit-rock interaction stands as a rather complex problem because the exact solution is very complicated to represent mathematically as they are formulated by delay differential equations. It is possible to model the rock axial forces as a spring-damper system that is dependent on formation properties and the system parameters like ROP and WOB. The numerical model representing bit-rock interaction includes the displacement component due to lifting action of the bit which is sinusoidal and with a frequency component which corresponds to the type of bit used [18].

For the purpose of this study, bit-rock interaction is represented by a sinusoidal wave with frequency equal to the frequency of rotation and different amplitude ranges. 20-sim parameter sweep is used to find the parametric effect of frequency and amplitude on the total system response.

\section{BOND GRAPH}

Bond graph is an interdisciplinary tool which helps in developing a dynamic model of a system with the help of the universal currency of physical systems, power. Its unique graphical nature makes it easy to visually observe the essential characteristics of the system [19]. Elements from various energy domains are represented with common energy storage, dissipating or transforming elements and are connected via

\begin{tabular}{|c|c|c|c|c|}
\hline \multicolumn{3}{|c|}{ Parameters } & Units & Values \\
\hline WOB & \multicolumn{2}{|c|}{ Weight } & $k N$ & $a+b \times n$ \\
\hline \multirow{4}{*}{$\begin{array}{l}\text { Drill } \\
\text { String }\end{array}$} & \multicolumn{2}{|c|}{ Length } & inch & 12 \\
\hline & \multicolumn{2}{|c|}{ ID } & inch & 0.75 \\
\hline & \multicolumn{2}{|c|}{ OD } & inch & 1 \\
\hline & \multicolumn{2}{|c|}{ Mass } & $\mathrm{kg}$ & 0.58 \\
\hline \multirow{8}{*}{$\begin{array}{l}\mathrm{p}-\mathrm{VARD} \\
\text { tool }\end{array}$} & \multirow{4}{*}{ 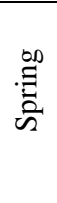 } & ID & inch & 0.755 \\
\hline & & OD & inch & 1.50 \\
\hline & & Thickness & inch & 0.045 \\
\hline & & Height & inch & 0.093 \\
\hline & \multirow{3}{*}{$\begin{array}{l}\dot{\bar{D}} \\
\stackrel{0}{0} \\
\vec{z}\end{array}$} & $\begin{array}{l}\text { Damping } \\
\text { ratio }(\zeta)\end{array}$ & - & $0.05-1.00$ \\
\hline & & $\begin{array}{l}\text { Damping } \\
\text { coefficient }\end{array}$ & $N s / m$ & $2 \zeta \sqrt{k m}$ \\
\hline & & $\begin{array}{l}\text { Number of } \\
\text { rubbers }\end{array}$ & - & 3 \\
\hline & \multicolumn{2}{|c|}{ Mass } & $k g$ & 10 \\
\hline \multirow{2}{*}{$\begin{array}{l}\text { Bit Rock } \\
\text { interaction }\end{array}$} & \multicolumn{2}{|c|}{ Frequency } & $r p m$ & $0-600$ \\
\hline & \multicolumn{2}{|c|}{ Amplitude } & $\mathrm{mm}$ & $0.5-2$ \\
\hline
\end{tabular}
power bonds.

Based on the premises developed in Section II, bond graphs are developed for the rigid and the compliant drilling systems with following parameters.

Table 1: Parameters of drill string system 
The bond graphs for the rigid and the compliant drilling system are shown in Figure 3 and Figure 4 respectively. In the bond graph, the sinusoidal displacement function is differentiated and fed into the system as the velocity input at the bit/rock contact point $(v 5)$. The p-VARD tool in Figure 4 is shown as a parallel combination of Belleville spring stack and rubber stack which is then connected to the drill string in series. The drill string in Figure 3 and Figure 4 is represented by a stiff spring with low damping and a one third lumped mass. Similarly, the velocity $v 1$ of top inertial component which generates the WOB is then integrated to observe the top displacement. All inertial components include sources of efforts (Se) due to the gravity.

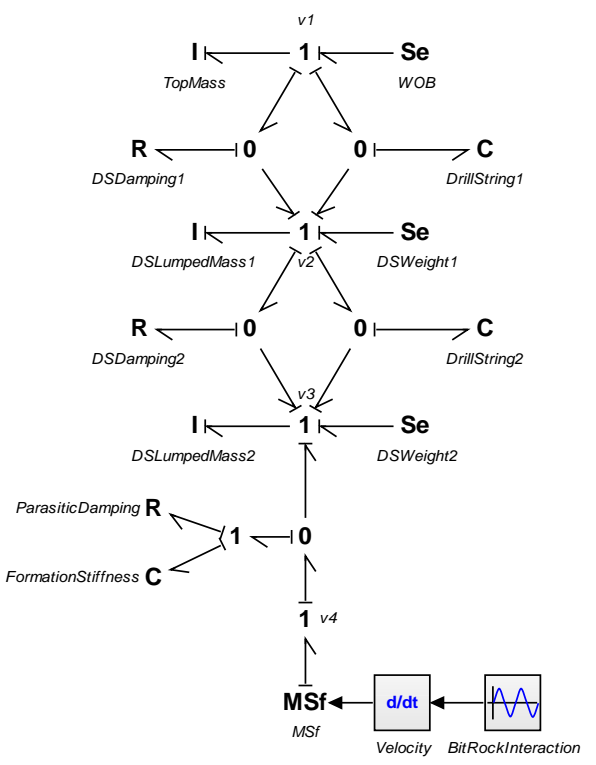

Figure 3: Bond graph model of a stiff drilling system

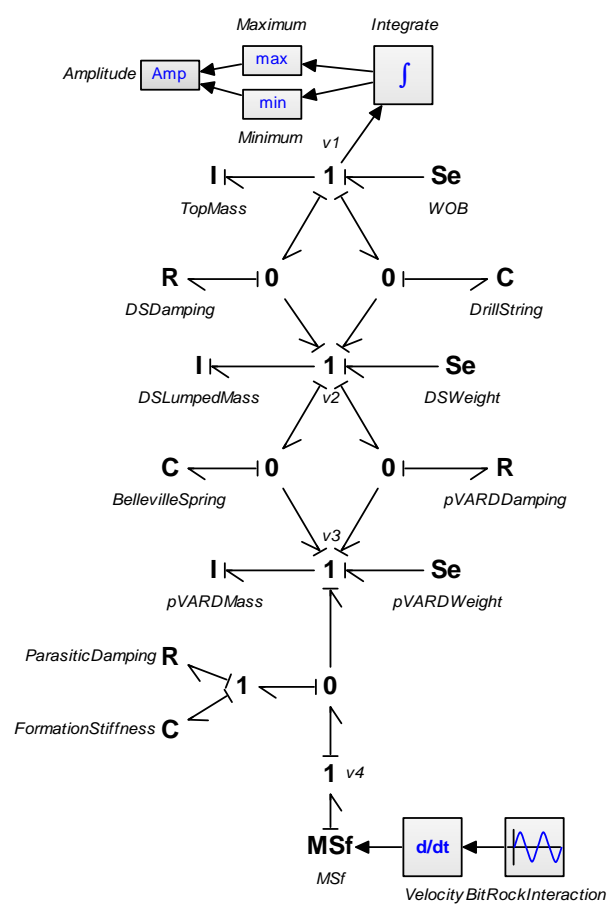

Figure 4: Bond graph model of a compliant drilling system

\section{RESUltS}

In this section, system parameters are varied, and their effects are observed. The primary effect of the p-VARD tool has been seen in softening the drill string and lowering its natural frequency. This can make the drill string resonate at lower workable ranges of frequencies comparable to typical drilling speeds. Similarly, another effect of p-VARD tool has been seen in the attenuation of excessive vibration particularly at frequencies higher than its natural frequency. In this section, system variables like damping ratio, excitation amplitude, compliance and WOB are plotted against the frequency of excitation followed by discussion and conclusion in Section V.

\section{A. Effect of Damping ratio( ()}

The damping ratio of the p-VARD tool is 0.05 , a standard value for Neoprene rubbers. This means that in one cycle, $5 \%$ of energy is dissipated by the system. To examine the effect of the damping ratio, its value is gradually increased from the underdamped condition of $\zeta=0.05$ to critically damped condition of $\zeta=1$ and the effects are observed. Figure 5 shows that the uncontrolled motion produced by the system resonance can be reduced by increasing the damping ratio and as the system approaches critical damping, the vibration amplification due to resonance becomes significantly low.

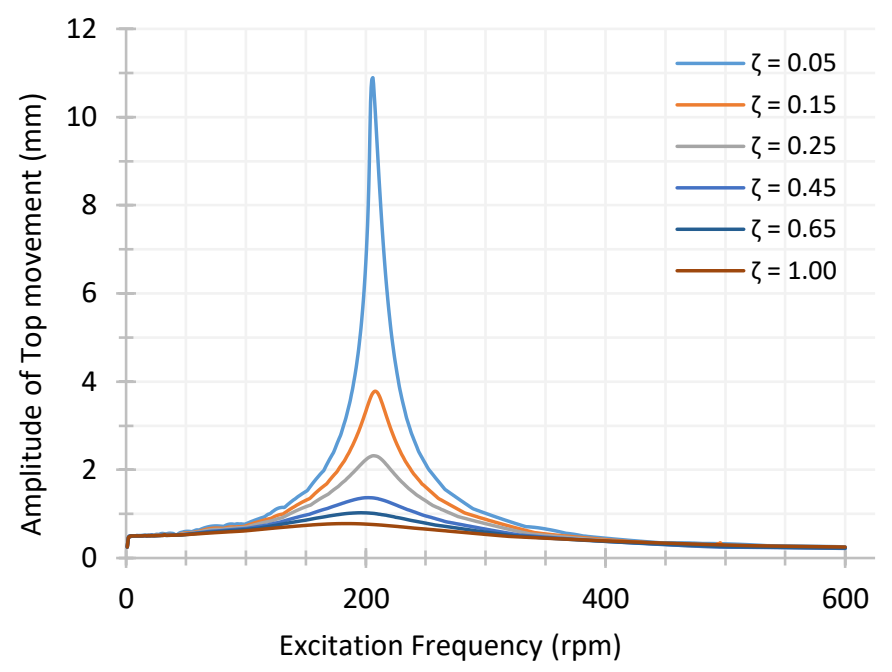

Figure 5: Showing the effect of damping ratio [System parameters: $I=20, J=1, a=0.5 \mathrm{~mm}]$

\section{B. Effect of excitation amplitude}

To observe the effect of the excitation amplitude, the damping ratio is set to $15 \%$ and the amplitude of vibration is gradually increased from $0.1 \mathrm{~mm}$ to $1.4 \mathrm{~mm}$. These parameters are selected because as the excitation frequency approaches the natural frequency of the system, the stack of Belleville springs tend to compress more frequently, and this phenomenon is more likely as amplitude of excitation increases. This limits the upper bound of excitation amplitude for a combination of spring configuration and damping ratio. One way to prevent the flattening of Belleville springs is to increase the damping ratio of the p-VARD tool as shown in Figure 5. As can be seen from Figure 6, the amplitude of excitation does not alter the range of system resonance, rather it scales up the magnitude of 
displacement of top mass. It is also observed that at amplitudes greater than $1.4 \mathrm{~mm}$, the Belleville springs become flat which instantaneously makes them stiff and stops the resonance.

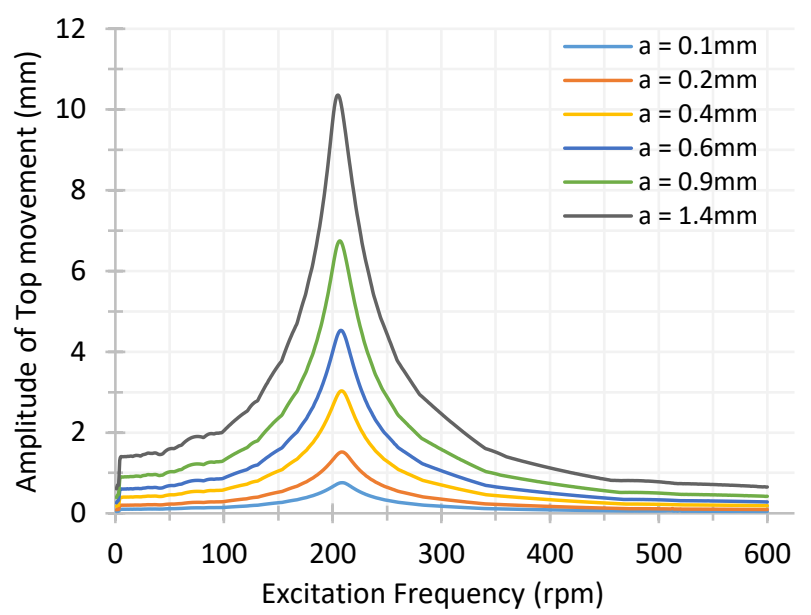

Figure 6: Showing the effect of excitation amplitude [System parameters: $I=20, J=1, \zeta=0.15]$

\section{Effect of $p$-VARD compliance}

The compliance of the p-VARD tool is altered by increasing/decreasing the number of springs in series and parallel. Increasing number of springs in series makes the springs more compliant while increasing number of springs in parallel makes them stiffer. As shown in Figure 7, it is observed that the resonating frequency of the system increases with the increase in p-VARD stiffness, which is justifiable as the natural frequency is directly proportional to the square root of the system stiffness. Also, because the p-VARD stiffness is less compared to that of the drill string, it can be assumed to be representative of the system stiffness.

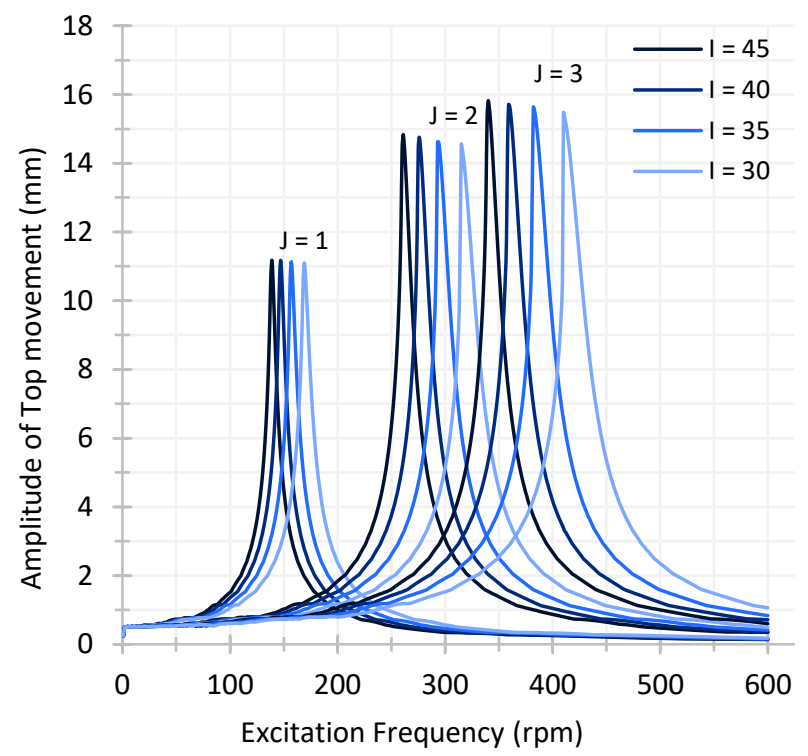

Figure 7: Showing the effect of spring compliance [System parameters: $\zeta=0.15$, excitation amplitude $=0.5 \mathrm{~mm}$ ]

\section{Effect of the Top Mass[WOB]}

The resonating frequency of a mass-spring-damper system is inversely proportional to the square root of the sprung mass, which in this case is primarily represented by the top mass, the gravitational force of which produces WOB of the system. The value of WOB is gradually increased by increasing the number of plates $(n)$ and the system response over the range of operating frequency is plotted. Figure 8 shows that there is a co-relation between the WOB and the resonating frequency, i.e. the system resonates at lower frequency as the WOB is increased.

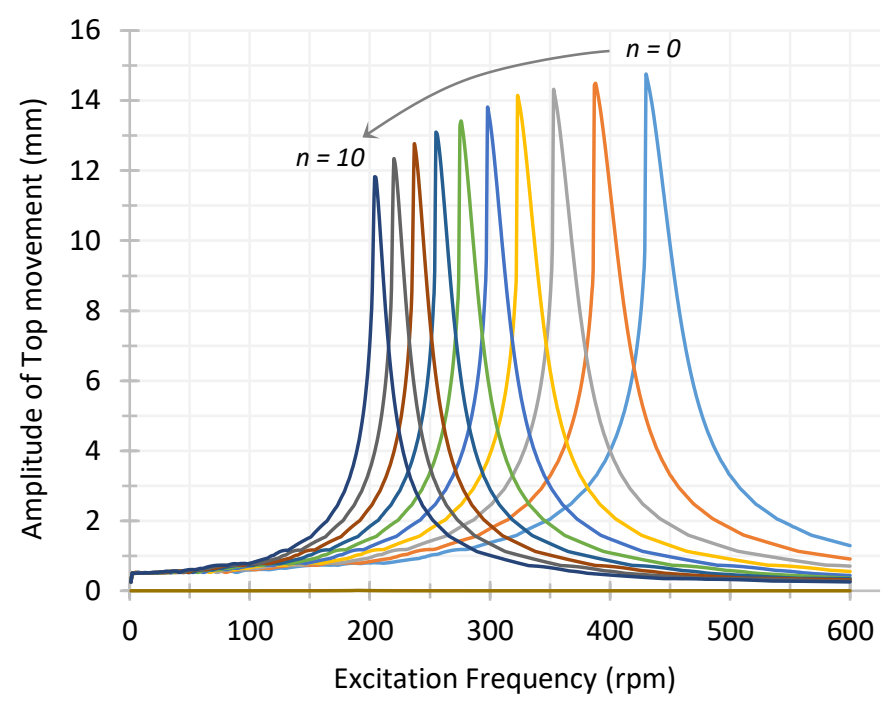

Figure 8: Showing the effect of WOB [System parameters: $I=25$, $J=2, \zeta=0.15$, excitation amplitude $=0.5 \mathrm{~mm}]$

\section{DisCUSSION AND CONCLUSION}

As seen from simulation results of Section IV, it is observed that adding a p-VARD tool to the drill string makes the whole system more compliant bringing the resonating frequency to a workable range. The system resonating frequency for a linear mass-spring-damper model of a rigid drill string system is at the range of $900 \mathrm{rad} / \mathrm{sec}$ or $9000 \mathrm{rpm}$ while adding a $\mathrm{p}$-VARD tool in series with the drill string can lower this frequency to the workable range of $300 \mathrm{rpm}$.

A study of the influence of system parameters of a compliant drilling system shows that spring configuration and weight on bit can alter the natural frequency of the drilling system. The lower the spring compliance, the higher is the resonating frequency and the higher the weight on bit, the lower is the resonating frequency. This can be observed from Figure 7 and Figure 8. In Figure 7, as the system becomes stiffer with more springs in parallel, the resonating frequency increases accordingly and as it becomes more compliant with more springs in series, the resonating frequency decreases. Similarly, in Figure 8 as the WOB is increased by increasing the number of plates, the system resonates at lower frequency. With reference to this plot, an operator can make the drill string resonate at desired frequency by altering the number of plates for a given configuration of Belleville springs, rubbers, drill string and rotary speed. 
Similarly, damping ratio of the rubber elements plays a critical role in attenuating the vibration. The damping ratio should be properly calibrated to prevent the flattening of Belleville springs, especially at around the system resonating frequency. Although the results due to insufficient damping are not included in this work, it will cause flattening of the springs and terminate the resonance prematurely. Of all, amplitude of excitation does not have significant effect in defining the nature of system response rather it only scales up or down the magnitude of vibration and does not alter the system natural frequency. However, higher amplitudes near the system natural frequency may cause flattening of springs.

The results from this study can help interpret the experimental data involving $\mathrm{p}-\mathrm{VARD}$ tool. As recorded in one of the experimental works by Molgaard et al. 2019 [8], the use of p-VARD tool showed the increase in recorded DDWOB. This increase can be assumed to be due to the resonance generated by the p-VARD tool. Figure 7 and Figure 8 show that the system can resonate successfully at $300 \mathrm{rpm}$ for different combination of system parameters. Similarly, this modeling can help in predicting the flattening of the Belleville springs due to high weight on bit or high excitation frequency. In this scenario, the stiffness of the system rises abruptly and reaches close to that of the drill string, which then stops the system excitation as the system natural frequency increases. A strategic design of experiment should avoid this phenomenon and let the system oscillate without flattening the springs. Also, flattening of Belleville springs may cause failure of torque transmitting keys and the outer shell of the p-VARD tool. However, care should be given to minimize the fatigue load generated by the high excitation amplitudes due to system resonance. This tradeoff between system oscillation and its fatigue life can be modelled and incorporated into the design of experiment, which can be a future work.

The relation between system parameters is consistent with that of a linear spring but use of bond graphs makes it easy to model the complicated load-deflection relationship of the Belleville spring. As an improvement to this work, modeling of frictional losses in Belleville springs can be done to understand the effect of spring lubrication and surface finishes.

\section{REFERENCES}

[1] W. C. Maurer, "The 'Perfect - Cleaning' Theory of Rotary Drilling," J. Pet. Technol., vol. 14, no. 11, pp. 1270-1274, 1962.

[2] T. M. Warren, "Penetration-Rate Performance of RollerCone Bits.," SPE Drill. Eng., vol. 2, no. 1, pp. 9-18, 1987.

[3] R. Teale, "The concept of specific energy in rock drilling," Int. J. Rock Mech. Min. Sci., vol. 2, no. 1, pp. 57-73, 1965.

[4] O. Oloruntobi and S. Butt, "Energy-based formation pressure prediction," J. Pet. Sci. Eng., vol. 173, no. October 2018, pp. 955-964, 2019.

[5] H. Li, S. Butt, K. Munaswamy, and F. Arvani, "Experimental investigation of bit vibration on rotary drilling penetration rate," 44th US Rock Mech. Symp. - 5th US/Canada Rock Mech. Symp., pp. 1-6, 2010.

[6] Y. Babatunde, S. Butt, J. Molgaard, and F. Arvani, "Investigation of the effects of vibration frequency on rotary drilling penetration rate using diamond drag bit," 45th US Rock Mech. / Geomech. Symp., 2011.

[7] M. Khademi, "Laboratory Study of the Effect of Axial Compliance on Rock Penetration of PDC Bits," Memorial University of Newfoundland, 2014.

[8] J. Molgaard, A. N. Abugharara, C. A. Hurich, and S. D. Butt, "Study of the influence of controlled axial oscillations of pvard on generating downhole dynamic wob and improving coring and drilling performance in shale," Proc. Int. Conf. Offshore Mech. Arct. Eng. OMAE, vol. 8, pp. 1-7, 2019.

[9] H. Khorshidian, S. D. Butt, and F. Arvani, "Influence of high velocity jet on drilling performance of PDC bit under pressurized condition," 48th US Rock Mech. / Geomech. Symp. 2014, vol. 1, pp. 343-348, 2014.

[10] S. Shah, Y. Xiao, and S. D. Butt, "Investigation of Drilling Performance in Core Bit Drilling using passive Vibration-Assisted Rotational Drilling (pVARD) Technology," 72nd Can. Geotech. Soc. Annu. Conf., no. 2011, 2019.

[11] "Pipinato, A. (Ed.). (2016). Innovative bridge design handbook: Construction, rehabilitation and maintenance. Retrieved from https://ebookcentral-proquest-com.qe2aproxy.mun.ca." .

[12] J. O. Almen and A. Laszlo, "The uniform-section disk spring," Trans. Am. Soc. Mech. Eng., vol. 58, pp. 305314, 1936.

[13] G. Curti and R. Montanini, "On the influence of friction in the calculation of conical disk springs," J. Mech. Des. Trans. ASME, vol. 121, no. 4, pp. 622-627, 1999.

[14] S. Ozaki, K. Tsuda, and J. Tominaga, "Analyses of static and dynamic behavior of coned disk springs: Effects of friction boundaries," Thin-Walled Struct., vol. 59, no. January, pp. 132-143, 2012.

[15] S. Shah, D. Maharjan, A. Abugharara, S. Imtiaz, S. Butt, and S. John, "Numerical and Experimental Study on Belleville Springs as Vibrational Including Element of Passive Vibration Assisted Rotary Drilling ( pVARD ) Tool for Drilling Performance Applications," in Proceedings of the Canadian Society for Mechanical Engineering, 2020.

[16] J. Zhong, "Investigation of Bit-Rock Interaction for Rotary Drilling and Influence on Penetration Rate," Memorial University of Newfoundland, 2015.

[17] "'Detailed Vibration Isolation Theory - Farrat Resources,' Farrat. [Online]. Available: https://www.farrat.com/resources/detailed-vibrationisolation-theory. [Accessed: 09-Mar-2020].," 2016. .

[18] F. Clayer, E. Aquitaine, J. K. Vandiver, and H. Y. Lee, "Effect of surface and downhole boundary conditions on the vibration of drillstrings," Proc. - SPE Annu. Tech. Conf. Exhib., vol. Delta, pp. 431-442, 1990.

[19] D. C. Karnopp, D. L. Margolis, and R. C. Rosenberg, System Dynamics, 5th ed. John Wiley \& Sons, Inc., 2017. 